\title{
DISTRIBUTION OF CHOLINERGIC VASOMOTOR NERVES TO THE PARAMETRIAL ARTERIES OF SOME LABORATORY AND DOMESTIC ANIMALS
}

\author{
C. BELL* \\ ARC Institute of Animal Physiology, Babraham, Cambridge
}

(Received 19th October 1970)

\begin{abstract}
Summary. The histochemical localization of acetylcholinesterase (AChE)-containing nerves has been determined in the parametrial arterial supplies of dog, cat, rat, rabbit, sheep, pig and cow.

The main uterine arteries of dog and pig contained a plexus of nerves which stained for AChE and which were closely applied to the surface of the media. This plexus was absent from the secondary arterial branches. In contrast, the equivalent arteries of rat, cat, rabbit, sheep and cow were devoid of an AChE-positive nerve plexus.

In the light of other evidence, it is suggested that the uterine hyperaemia of pregnancy in dog and pig may be partly due to the action of cholinergic vasodilator nerves. Such a mechanism is unlikely to be involved in the other species examined.
\end{abstract}

\section{INTRODUCTION}

Pregnancy constitutes a unique cardiovascular situation in that it demands a massive and maintained increase in regional blood flow in order to satisfy the metabolic demands of the growing foetus. This adjustment is partly achieved by hypertrophy of the uterine vessels, and the morphological details of this phenomenon have been described for a variety of species (Barcroft \& Barron, 1946; Reynolds, 1949, 1963; Orsini, 1957; Ramsey, 1959). It is not established, however, whether there is an active dilator component to the hyperaemic condition.

Recently, it was reported that the extrinsic uterine arterial supply of the guinea-pig is innervated by cholinergic vasodilator nerves which are functional only during pregnancy and it has been suggested that these nerves are involved in the production of uterine hyperaemia in this species (Bell, 1968, 1969a). In the present investigation, the extrinsic uterine arteries of some other common mammals have been examined histochemically and differences have been revealed in the pattern of innervation which indicate that the experimental species used may be an important factor for preclinical studies of uterine blood flow.

* Present address: Department of Zoology, University of Melbourne, Parkville, Victoria 3052, Australia. 


\section{METHODS}

The following animals were used: eight rats (Wistar), five cats (crossbred), five rabbits (New Zealand white), four dogs (crossbred), four sheep (one Soay, three Clun Forest), four pigs (Large White), three cows (assorted breeds).

Animals were killed by cervical fracture (rat, rabbit), by captive-bolt pistol (pig, sheep, cow) or by bleeding under anaesthesia. The uterus, together with the extrinsic vasculature running in the broad ligament, was removed and placed in $0.9 \% \mathrm{w} / \mathrm{v}$ saline. Small pieces of ileum, and on occasions skeletal muscle, were also removed. The extrinsic uterine vessels were cleaned of any surrounding adipose tissue and fixed at in vivo length overnight at $6^{\circ} \mathrm{C}$ in $10 \%$ formol sucrose buffered to $\mathrm{pH} 6.7$ with $\mathrm{NH}_{3}$ (Pearson, 1963).

Following fixation, the tissues were washed for several hours in at least three changes of distilled water and segments, or $15 \mu \mathrm{m}$ frozen transverse sections, of main and secondary uterine arteries were stained for acetylcholinesterase (AChE) by the method of Karnovsky \& Roots (1964). Acetylthiocholine substrate was used, for the minimum period producing satisfactory resolution of known cholinergic nervous elements in similarly prepared samples of ileum and skeletal muscle from the same species. In some experiments, 1:5-bis(4-allyldimethylammoniumphenyl)pentane-3-one-diiodide (BW284C51) $\left(5 \times 10^{-6} \mathrm{M}\right)$ or physostigmine sulphate $\left(10^{-5} \mathrm{M}\right)$ were employed as inhibitors of AChE and total cholinesterase activity, respectively. After staining, the tissues were washed in distilled water for at least $10 \mathrm{~min}$. Frozen sections were mounted on slides coated with albumin, dehydrated, cleared and mounted in Xam. Vessel segments were embedded in paraffin wax and sectioned at 8 to $10 \mu \mathrm{m}$, or dehydrated, cleared and mounted whole.

\section{RESULTS}

\section{Dog and pig}

In dog and pig, the main uterine artery was accompanied by a plexus of fine nerve fibres which stained heavily and which were applied closely to the outer surface of the media (Pl. 1, Figs. 1, 3). In contrast, no such staining fibres were observed in the secondary arterial branches running to the uterine horns ( $\mathrm{Pl}$. 1, Figs. 2, 4). In both species, the arterial supply was also accompanied by a variable number of large, heavily stained nerve trunks which ran in the outer arterial adventitia and were probably supplying the uterus itself. All staining

\section{EXPLANATION OF PLATE 1}

Sections of parametrial uterine arteries stained for AChE. During photography, the microscope condenser has been slightly closed to show up the unstained vessel walls. Calibration line on each figure $=100 \mu \mathrm{m}$.

FIG. 1. Dog: main uterine artery. Note the staining perivascular nerve plexus (X) and scattered staining bundles (B).

FIG. 2. Dog: secondary uterine artery. Although staining nerve bundles still accompany the artery $(B)$, the perivascular plexus is absent at the medio-adventitial border (arrow). Frg. 3. Pig: main uterine artery. Note the plexus of staining nerve fibres at the medioadventitial border $(\mathrm{X})$.

FIG. 4. Pig: secondary uterine artery. The staining plexus is now absent at the medioadventitial border (arrow). Some staining nerve bundles accompany the artery (B). 
PLATE 1
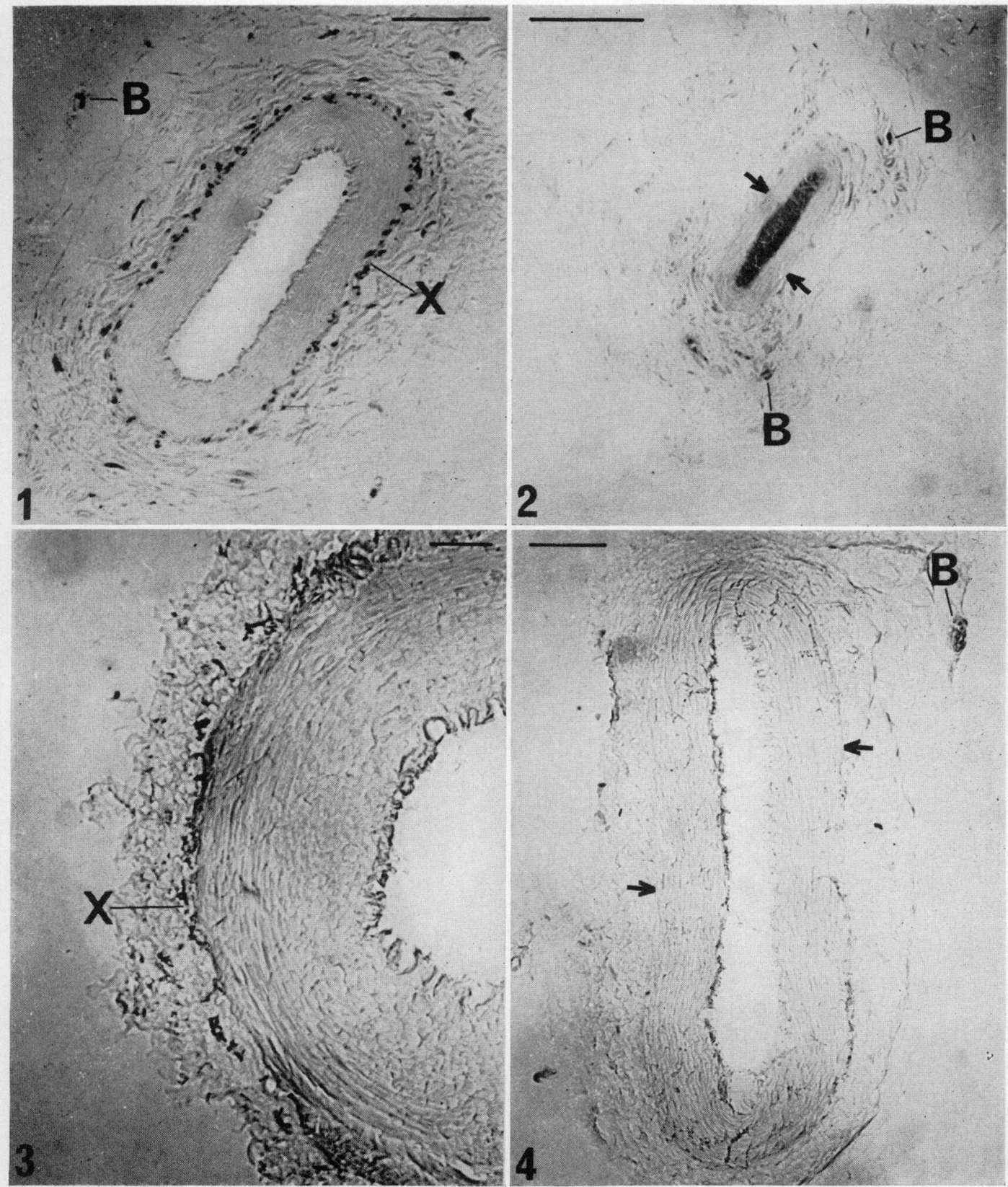

(Facing p. 54) 
PLATE 2

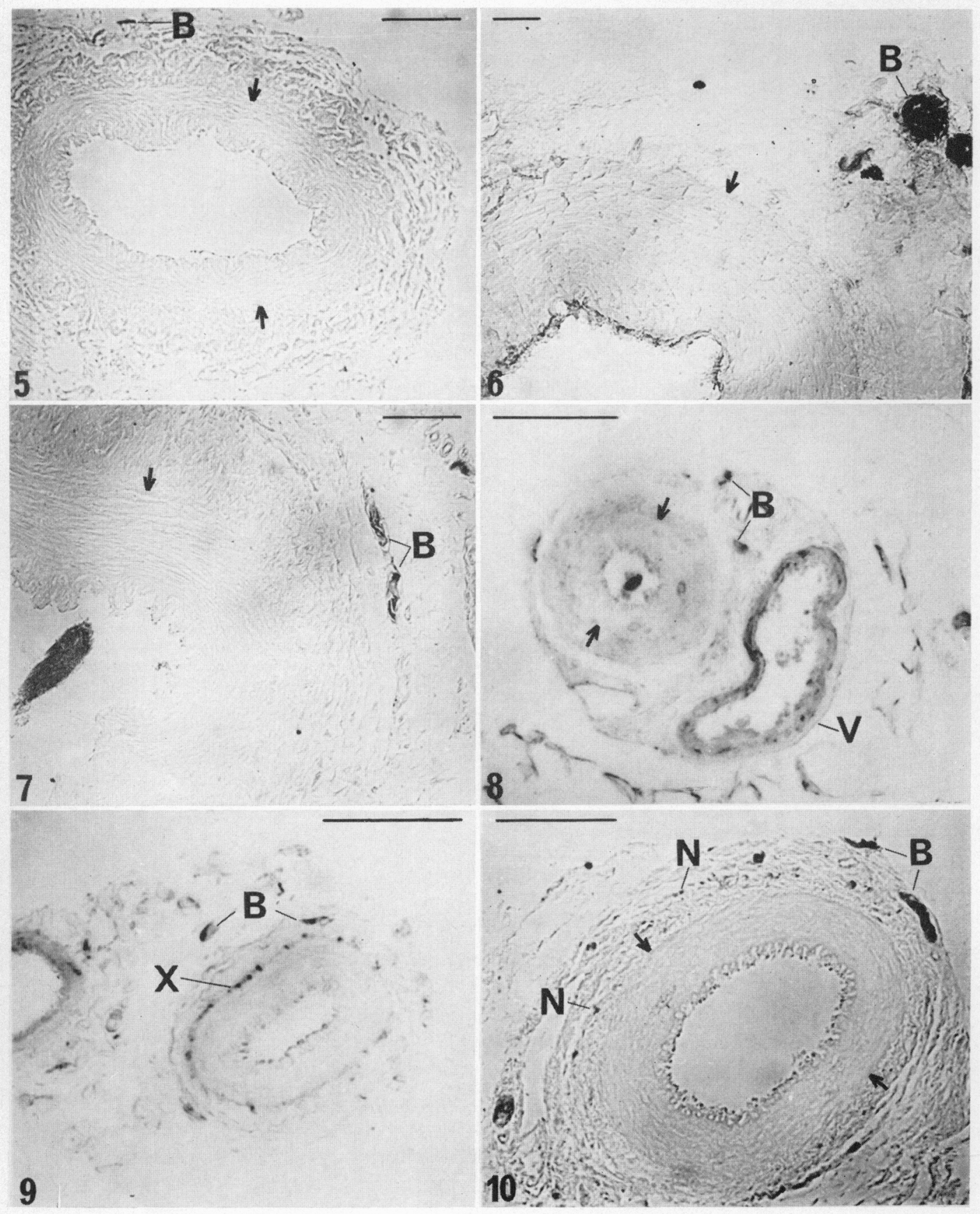

(Facing p. 55) 
was prevented by treatment of the tissue with BW284C51, indicating that the reaction was due entirely to the presence of AChE (Austin \& Berry, 1953; Holmstedt, 1957).

\section{Cat, sheep and cow}

In these species, no staining nerve plexus was seen to accompany either the main (in the sheep both caudal and medial vessels were examined) or the secondary arteries (PI. 2, Figs. 5 to 7). However, the vessels were accompanied by a variable number of large, heavily stained, nerve trunks which lay in the outer arterial adventitia. The staining reactivity of these trunks was prevented in tissues which had been treated with BW284C51, indicating that staining was due entirely to AChE.

TABLE 1

DISTRIBUTION OF AGhE-POSITIVE NERVES RUNNING WITH THE PARAMETRIAL UTERINE VASGULATURE OF VARIOUS ANIMAL SPEGIES

\begin{tabular}{|c|c|c|c|}
\hline Species & $\begin{array}{r}\text { Perivasculo } \\
\text { Main uterine artery }\end{array}$ & $\begin{array}{l}\text { plexus } \\
\text { Secondary branches }\end{array}$ & $\begin{array}{c}\text { Perivascular nerve } \\
\text { trunks }\end{array}$ \\
\hline $\begin{array}{l}\text { Guinea-pig* } \\
\text { Human } \dagger \\
\text { Dog } \\
\text { Pig } \\
\text { Rat } \\
\text { Rabbit } \\
\text { Cat } \\
\text { Sheep } \\
\text { Cow }\end{array}$ & $\begin{array}{l}+++ \\
+++ \\
+++ \\
+++ \\
+1- \\
+1- \\
- \\
-\end{array}$ & $\begin{array}{l}- \\
- \\
- \\
- \\
- \\
- \\
- \\
-\end{array}$ & $\begin{array}{l}\text { Yes } \\
\text { Yes } \\
\text { Yes } \\
\text { Yes } \\
\text { Yes } \\
\text { Yes } \\
\text { Yes } \\
\text { Yes } \\
\text { Yes }\end{array}$ \\
\hline
\end{tabular}

* Bell (1968).

$\dagger$ Bell (1969b).

$(-)$, Nerves absent; $(+)$, few nerves present; $(+++)$, many nerves present.

\section{Rat and rabbit}

As in the other species examined, the parametrial arteries in rat and rabbit were accompanied by large, heavily stained nerve trunks. In two of the eight rats examined, the medio-adventitial junction of the main uterine artery con-

\section{EXPLANATION OF PLATE 2}

Calibration line on each figure $=100 \mu \mathrm{m}$.

Fig. 5. Cat: main uterine artery. Note absence of staining fibres at the medio-adventitial border (arrow).

FIG. 6. Cow: main uterine artery. A staining nerve trunk is visible (B), but the medioadventitial border is devoid of a staining nerve plexus (arrow).

FIG. 7. Sheep: main uterine artery. Two staining nerve trunks are visible (B), but the medio-adventitial border is devoid of a staining nerve plexus (arrow).

FIG. 8. Rat: main uterine artery, with accompanying vein $(\mathrm{V})$ and staining nerve bundles $(B)$. Note the absence of a staining nerve plexus at the medio-adventitial border (arrow).

FIG. 9. Rat: main uterine artery, as in Fig. 8. In this animal, however, a moderate population of staining nerve fibres lying at the medio-adventitial border was apparent (X). Fic. 10. Rabbit: main uterine artery. Numerous staining nerve bundles $(B)$ accompanying the vessel. There is no staining nerve plexus visible in the region of the medio-adventitial border (arrows), although the adventitia contains some small staining nerves (N). 
tained a moderate number of small heavily stained fibres. In the remaining six animals, the arteries were completely devoid of any staining fibres except for those in the large nerve trunks (Pl. 2, Figs. 8, 9). In the rabbit, no staining fibres were seen at the medio-adventitial junction. However, there were a few small, heavily stained nerve bundles scattered through the adventitia of the main uterine artery (Pl. 2, Fig. 10). In both rat and rabbit, the staining reaction obtained was completely prevented in tissues which had been treated with physostigmine, but was only reduced in intensity by about $50 \%$ in tissues treated with BW284C51, suggesting the presence of cholinesterase activity other than that due to AChE. The existence of comparatively large amounts of 'pseudo'-cholinesterase has been remarked previously in the nervous systems of both rat (Koelle, 1954; Shute \& Lewis, 1963) and rabbit (Koelle, 1955).

The above findings are summarized in Table 1.

\section{DISCUSSION}

It has become apparent from a growing number of histochemical and electron microscopic studies that a plexus of fine nerve fibres closely associated with the surface of the media of a blood vessel represents a true vasomotor innervation, as distinct from the larger nerve bundles which may be situated in the outer adventitia and travel with the vessel to more distant effectors (Hillarp, 1959; Norberg \& Hamberger, 1965; Bell, 1969a). The present study has shown such a plexus of AChE-positive nerves to exist in the main uterine arteries of the dog and the pig. It may therefore be assumed that these nerves represent a vasomotor innervation of the arteries. Such a conclusion is supported by the fact that the plexus does not extend to the secondary branches of the artery and, therefore, cannot be regarded as a population of nerves travelling with the vessels to the uterus itself. This plexus exhibited an intensity of AChE reactivity similar to that seen in known cholinergic elements from the same species, suggesting that it was composed of cholinergic nerves. It has been demonstrated previously for both the dog (Jacobowitz \& Nemir, 1969; Fillenz, 1970; Bell \& McLean, 1970) and for the pig (Hebb, 1969) that the level of AChE in cholinergic autonomic nerves is much greater than that in adrenergic nerves.

The presence of this apparent cholinergic vasomotor nervous supply to the main, but not to the secondary uterine arteries in dog and pig is similar to the pattern of innervation in the guinea-pig (Table 1). In the guinea-pig (Bell, 1968), it has been shown that this innervation, which is a dilator, is functional only during pregnancy, and it has been suggested that it is involved in the concomitant increase of uterine blood flow (Bell, 1969a). The existence during pregnancy in the guinea-pig of active cholinergic dilatation of the parametrial arteries has more recently been confirmed by arteriographic techniques (Bell \& Brown, 1971). It seems likely, therefore, that the plexuses in the dog and pig are also dilators and may be concerned with the production of uterine hyperaemia of pregnancy. It is of interest that sacral parasympathectomy during pregnancy in the dog has been reported to cause a pronounced fall in placental blood flow, suggesting the existence of a neurogenic dilatory mechanism (Abou-Shabanah, Ullery \& Wenger, 1958; Toth, McEwen \& Shabanah, 1964). 
In contrast to the situation in the above species, medio-adventitially located nerves staining heavily for $\mathrm{AChE}$ were sparse or absent in the uterine arteries of rat, rabbit, cat, sheep and cow. Thus, neurogenic dilatation, at least of a cholinergic nature, is unlikely to be concerned with uterine hyperaemia of pregnancy in these species. Physiological evidence concerning other possible mechanisms is lacking for most species. However, blood flow measurements in vivo in the sheep have supported the view that neurogenic influences are absent and have suggested that there may be a direct dilator influence exerted by circulating hormones (Greiss, Gobble, Anderson \& McGuirt, 1967).

The extrinsic uterine arteries of the human (Bell, 1969b) have been shown to receive a cholinergic vasomotor innervation with a similar distribution to that seen in guinea-pig, dog and pig (Table 1). In view of the striking interspecies difference in the pattern of uterine arterial innervation, it seems that the guineapig, dog and pig may be more suitable than some other commonly used experimental animals for studies of uterine blood flow intended to have clinical relevance.

\section{ACKNOWLEDGEMENTS}

I should like to thank Dr M. Vogt for her hospitality and for her interest in this work, and the National Heart Foundation of Australia for personal support as an Overseas Research Fellow. I am also grateful to Mr I. S. King for technical assistance, and to Mr G. A. Embleton, Speechley's Abbatoirs and my colleagues at Babraham for making available some of the animals used.

\section{REFERENCES}

Abou-Shabanah, E. H., Ullery, J. C. \& Wenger, J. B. (1958) A phase of the autonomic nervous system in obstetrics and gynecology. Surg. Gynec. Obstet. 107, 744.

Austin, L. \& BerRy, W. K. (1953) Two selective inhibitors of cholinesterase. Biochem. J. 54, 695.

BARcroft, J. \& BARron, D. H. (1946) Observations upon the form and relations of the maternal and fetal vessels in the placenta of the sheep. Anat. Rec. 94, 569.

Bell, G. (1968) Dual vasoconstrictor and vasodilator innervation of the uterine arterial supply in the guinea-pig. Circulat. Res. 23, 279.

Bell, G. (1969a) Fine structural localization of acetylcholine-esterase at a cholinergic vasodilator nerve-smooth muscle synapse. Circulat. Res. 24, 61.

BeLl, C. (1969b) Evidence for dual innervation of the human extrinsic uterine arteries. 7. Obstet. Gynaec. Br. Commonw. 76, 1123.

Bell, C. \& Brown, M. J. (1971) Arteriographic evidence for a cholinergic dilator mechanism in uterine hyperaemia of pregnancy in the guinea-pig. 7. Reprod. Fert. 26, 59.

BELI, C. \& MCLEAN, J. R. (1970) The distribution of cholinergic and adrenergic fibres in the retractor penis and vas deferens of the dog. Z. Zellforsch. mikrosk. Anat. 106, 516.

Fillenz, M. (1970) Innervation of pulmonary and bronchial blood vessels of the dog. F. Anat., Lond. $106,449$.

Greiss, F. G., Jr, Gobble, F. L., Jr, Anderson, S. G. \& MaGuirt, W. F. (1967) Effect of acetylcholine on the uterine vascular bed. Am. J. Obstet. Gynec. 99, 1073.

HesB, C. O. (1969) Motor innervation of the pulmonary blood vessels of mammals. In: The Pulmonary Circulation and Interstitial Space, p. 195. Eds. A. P. Fishman and H. H. Hecht. University of Chicago Press.

Hillarp, N.- $\AA$ (1959) The construction and functional organization of the autonomic innervation apparatus. Acta physiol. scand. 46, Suppl. 157.

Holmstedt, B. (1957) A modification of the thiocholine method for the determination of cholinesterase. I. Biochemical evaluation of selective inhibitors. Acta physiol. scand. 40, 322.

Jacoвowtтz, D. \& NemiR, P. (1969) The autonomic innervation of the esophagus of the dog. 7. thorac. cardiovasc. Surg. 58, 678. 
Karnovsky, M. J. \& Roots, L. (1964) A 'direct-coloring' thiocholine method for cholinesterases. F. Histochem. Cytochem. 12, 219.

Koelze, G. B. (1954) The histochemical localization of cholinesterases in the central nervous system of the rat. F. comp. Neurol. 100, 211.

Koelle, G. B. (1955) The histochemical identification of acetycholinesterase in cholinergic, adrenergic and sensory neurons. F. Pharmac. exp. Ther. 114, 167.

Norberg, D.-A. \& Hamberger, B. (1965) The sympathetic adrenergic neuron. Some characteristics revealed by histochemical studies on the intraneuronal distribution of the transmitter. Acta physiol. scand. 63, Suppl. 238.

OrsinI, M. U. (1957) The vascular knot of the hamster uterus; the placental arterial supply and its changes during gestation and postpartum involution. 7 . Morph. 100, 565.

Pearson, C. K. (1963) A formalin-sucrose ammonia fixative for cholinesterases. F. Histochem. Cytochem. 11,665 .

Ramsex, E. M. (1959) Vascular adaptations of the uterus to pregnancy. Ann. N.Y. Acad. Sci. 75, 726.

ReYnolds, S. R. M. (1949) Physiology of the uterus, 2nd edn. Hoeber, New York.

Reynolos, S. R. M. (1963) Maternal blood flow in the uterus and placenta. In: Handbook of Physiology, Circulation, Vol. 2, p. 1585. Am. Physiol. Soc., Washington.

Shute, C. C. D. \& LEWIs, P. R. (1963) Cholinesterase-containing systems of the brain of the rat. Nature, Lond. 199, 1160.

Toth, A., McEwen, R. \& Shabanah, E. H. (1964) Role of the autonomic nervous system in the nutrition of the products of conception: effect of pelvic parasympathectomy on uterine and subplacental decidual blood flow. Fert. Steril. 15, 263. 